\title{
MeCP2 controls hippocampal brain-derived neurotrophic factor expression via homeostatic interactions with microRNA-132 in rats with depression
}

\author{
MEILEI SU, JUN HONG, YONGZHI ZHAO, SHUAI LIU and XIANG XUE \\ Department of Psychology, Nanfang Hospital, Southern Medical University, \\ Guangzhou, Guangdong 510515, P.R. China
}

Received August 18, 2014; Accepted June 3, 2015

DOI: $10.3892 / \mathrm{mmr} .2015 .4104$

\begin{abstract}
Major depressive disorder (MDD) is a considerable public health concern, which affects patients worldwide. MDD is associated with psychosocial impairment, poor quality of life, and significant disability, morbidity and mortality. Stress is a major factor in depression, which impairs the structural and functional plasticity of the hippocampus. Previous studies have demonstrated that chronic unpredictable mild stress is able to downregulate the expression of brain-derived neurotrophic factor (BDNF) and methyl-CpG-binding protein 2 (MeCP2), and alter the expression levels of certain microRNAs (miR). The aim of the present study was to investigate the regulatory association between BDNF, MeCP2 and miR-132 in an animal model of chronic stress-induced depression. ELISA, western blot and qPCR were used to detect the expression levels of BDNF, MeCP2 and miR-132 in the peripheral blood samples of patients with MDD and in the hippocampi of depressed animals. In addition, a dual luciferase reporter gene system was used to determine whether miR-132 directly targets $\mathrm{BDNF}$ or MeCP2. The present study demonstrated that, as compared with normal subjects, miR-132 expression was increased in the peripheral blood samples of patients with MDD, whereas the expression of MeCP2 and BDNF was decreased; thus, the expression levels of MeCP2 and BDNF were negatively correlated with those of miR-132. In addition, in an animal model of chronic stress-induced depression, increased expression levels of miR-132, and decreased levels of MeCP2 and BDNF were detected in the hippocampi. Furthermore, knockdown of MeCP2 expression
\end{abstract}

Correspondence to: Ms. Meilei Su, Department of Psychology, Nanfang Hospital, Southern Medical University, 1838 Guangzhou Avenue North, Guangzhou, Guangdong 510515, P.R. China E-mail: sumeilei@126.com

Key words: major depressive disorder, brain-derived neurotrophic factor, methyl-CpG-binding protein 2, microRNA, homeostatic interaction in primary hippocampal neurons increased the expression of miR-132 and decreased the expression levels of BDNF. The results of the present study demonstrated that miR-132 may directly target MeCP2, but not BDNF, and control its expression at the transcriptional and translational level. miR-132 was also shown to negatively regulate BDNF expression. The reduced expression levels of BDNF, as induced by MeCP2 knockdown, were enhanced by miR-132 mimics, and were rescued by miR-132 inhibitors. These results suggested that homeostatic interactions between MeCP2 and miR-132 may regulate hippocampal BDNF levels, which may have a role in the pathogenesis of MDD.

\section{Introduction}

Major depressive disorder (MDD) is a considerable public health concern, which affects patients worldwide. MDD is associated with psychosocial impairment, poor quality of life, and significant disability, morbidity and mortality (1). However, due to the poor understanding regarding the pathogenic mechanisms associated with MDD, $40 \%$ of patients with MDD do not respond well to the currently available treatments (2). A previous study in patients with MDD detected neuronal atrophy, altered dendritic morphology of hippocampal neurons and decreased hippocampal volume (3).

Stress is a major factor in depression, which impairs the structural and functional plasticity of the hippocampus (4). Chronic and persistent stress is able to induce dysfunction of the hypothalamic-pituitary-adrenal axis, alter the immune system and induce other pathophysiological effects detected in patients with depression (5). A previous study demonstrated that chronic unpredictable mild stress is able to downregulate the expression of brain-derived neurotrophic factor (BDNF), which has a critical role in the etiology of depression; the downregulation of which is reversed following classical antidepressant therapy (6). Furthermore, emerging evidence has demonstrated that microRNAs (miRNAs) are associated with depression, particularly miRNAs involved with BDNF (7). miRNA alterations have been detected in patients with MDD and rats exposed to chronic stress (8). miRNAs are a class of short ( $22 \mathrm{nt})$ non-coding RNAs that suppress the expression of various genes at the post-transcriptional level by targeting 
the $3^{\prime}$ untranslated region (3'UTR). miRNAs are essential for numerous cellular processes, including survival, differentiation and apoptosis, in humans and animals $(9,10)$.

miRNAs are regulated by specific upstream molecules. Epigenetic mechanisms are well-known mechanisms that regulate the expression of miRNAs $(11,12)$. Methyl-CpG-binding protein $2(\mathrm{MeCP} 2)$ is a transcription factor that binds to methylated cytosine residues on $\mathrm{CpG}$ dinucleotides in DNA, resulting in the recruitment of histone deacetylases and other transcriptional repressors that silence target genes (13). A previous study demonstrated that the loss of MeCP2 function causes Rett syndrome (14). In addition, it has been suggested that MeCP2 may have a critical role in depression (15). Murgatroyd et al (16) demonstrated that neuronal activity controlled the ability of $\mathrm{MeCP} 2$ to regulate activity-dependent transcription of the arginine vasopressin gene and induced epigenetic marking. Hutchinson et al (15) demonstrated that $\mathrm{MeCP} 2$ phosphorylation was required for the beneficial effects of chronic imipramine treatment on chronic social defeat stress-induced depressive-like behaviors. In addition, miRNAs may decrease MeCP2 expression levels in human gastric carcinoma cell lines and in cultured mouse cortical neurons $(17,18)$. Notably, $\mathrm{MeCP} 2$ is able to regulate cocaine intake through controlling the effects of cocaine on striatal BDNF levels by interacting with miR-212. (19). Therefore, the present study hypothesized that interactions between MeCP2 and miRNAs may have a role in depression.

The aim of the present study was to investigate the regulatory association between $\mathrm{BDNF}, \mathrm{MeCP} 2$ and miR-132 in vitro and in vivo. The expression levels of $\mathrm{BDNF}, \mathrm{MeCP} 2$ and miR-132 were detected in peripheral blood samples obtained from patients with MDD (using ELISA, western blotting and reverse transcription-quantitative polymerase chain reaction; RT-qPCR), and in the hippocampi of depressed rats. In addition, the regulatory association between BDNF, MeCP2 and miR-132 was determined using a dual luciferase reporter gene system, as well as with gain- and loss-function experiments.

\section{Materials and methods}

Blood samples. A total of 60 blood samples were collected from patients with MDD $(n=30)$ and age-matched normal subjects $(n=30)$ without other severe diseases, including diabetes, epilepsy and dementia, from Nanfang Hospital, Southern Medical University (Guangzhou, China). Written informed consent was obtained from the patients. In addition, the present study was approved by the ethical committee of the Southern Medical University. All samples were collected according to the legislation and ethical boards of Nanfang Hospital. All of the samples were stored at $-80^{\circ} \mathrm{C}$ until further use.

Animals. Male Sprague-Dawley rats, (age, 2 months; weight, $\sim 220 \mathrm{~g}$ ) obtained from Geneseed Biotech Co.,Ltd. (Guangzhou, China) were housed in groups of four per cage. The animals were allowed 1 week of habituation after arrival prior to stress exposure. All of the rats were housed in standard conditions (12 h light/dark, $25 \pm 1^{\circ} \mathrm{C}, 50 \%$ humidity) with controlled access to food and water. All of the animal procedures were performed in accordance with the National Institutes of Health Guide for the Care and Use of Laboratory Animals, and the procedures were approved by the Local Animal Use Committee of the Southern Medical University.

Cell culture and treatment. E18 rat fetuses (Geneseed Biotech Co., Ltd.) were used to prepare primary hippocampal neurons. Hippocampi were mechanically dissociated from the brains of the fetuses and treated with trypsin (Sigma-Aldrich, St. Louis, MO, USA) in phosphate-buffered saline (PBS) for $15 \mathrm{~min}$ at $37^{\circ} \mathrm{C}$. The cell suspensions were maintained in glial-conditioned medium (Invitrogen Life Technologies, Carlsbad, CA, USA) in $100-\mathrm{mm}$ dishes at $37^{\circ} \mathrm{C}$ in an atmosphere containing $5 \% \mathrm{CO}_{2}$ until further use. Ectopic cellular expression of miR-132 was achieved by transfection with miR-132 mimics or inhibitors (cat. nos. miR10000838-1-5 and miR20000838-1-5, respectively; Guangzhou RiboBio Co., Ltd., Guangzhou, China) using Lipofectamine ${ }^{\circledR} 2000$ (Invitrogen Life Technologies) for $48 \mathrm{~h}$, according to the manufacturer's instructions. Following treatment with the demethylation drug 5-Aza-2'-deoxycytidine (Aza; $15.55 \mathrm{nM}$; Sigma-Aldrich) for $72 \mathrm{~h}$, the miR-132 expression in the primary hippocampal neurons was detected using qPCR. For MeCP2 knockdown, a 70 nt short hairpin RNA (shRNA) was designed by GenScript Co., Ltd. (Nanjing, China) and pRNA-U6.1/Neo/CTL (cat. no. SD1801; GenScript Co., Ltd.) served as the control shRNA. The shRNA was cloned into the pRNAT-U6.2/Lenti expression vector by GenScript Co., Ltd. and the vector was transfected into primary hippocampal neurons. Control vectors were identical to the expression constructs without the gene insert.

CUS exposure. The rats were exposed to a variable sequence of mild and unpredictable stressors for 28 days. The rats were exposed to two random stressors per day, out of 10 various stressors. The stressors included a forced cold $\left(4^{\circ} \mathrm{C}\right)$ swim for $5 \mathrm{~min}$, food deprivation for $24 \mathrm{~h}$, water deprivation for $24 \mathrm{~h}$, light/dark cycle reversal for $36 \mathrm{~h}$, vibration for $1 \mathrm{~h}$, cage tilting for $24 \mathrm{~h}$, cold $\left(4^{\circ} \mathrm{C}\right)$ for $1 \mathrm{~h}$, crowding for $24 \mathrm{~h}$, soiled bedding for $24 \mathrm{~h}$, and tail clamp for $1 \mathrm{~min}$.

Sucrose preference test. The sucrose preference test is used to detect anhedonia. Prior to the experiment, the rats were trained to adapt to a $1 \%$ sucrose solution (w/v) for $48 \mathrm{~h}$. Following water deprivation for $4 \mathrm{~h}$, the rats were housed in individual cages for $4 \mathrm{~h}$, which contained two identical bottles, one filled with $1 \%$ sucrose solution and the other filled with water. At the end of the 4-h test, sucrose and water consumption (g) was measured. Sucrose preference (\%) was calculated using the following formula: Sucrose preference $(\%)=$ sucrose consumption/(sucrose consumption + water consumption). The less sucrose that was consumed, the more severe the case of anhedonia.

Forced swim test (FST). One day prior to the experiment, each rat was individually placed into a plastic cylinder (diameter, $25 \mathrm{~cm}$; height, $55 \mathrm{~cm})$ filled with water $\left(23-25^{\circ} \mathrm{C}\right)$ to a depth of $45 \mathrm{~cm}$ for $15 \mathrm{~min}$. The rats were subsequently removed from the water and returned to their cages. After $24 \mathrm{~h}$, a 5 min FST was performed. The FST was performed as previously described (20). Immobility time (in sec) was recorded by two independent observers. Floating was defined as the 
minimum movement necessary to maintain the heads of the rats above the water. The percentage of immobility time (\%) was calculated using the following formula: Immobility time $(\%)=$ immobility time/total experimental time.

$R T$ - $q P C R$. An Ultrapure RNA kit (Beijing CWBiotech Co., Ltd., Beijing, China) was used to extract total RNA from the primary hippocampal neurons, which were collected by trypsinization, according to the manufacturer's instructions. RNA was reverse transcribed to cDNA using a RevertAid First Strand cDNA Synthesis kit (Thermo Fisher, Inc., Waltham, MA, USA) according to the manufacturer's instruction. The cDNA $(2 \mu \mathrm{g})$ was then used to perform qPCR with a CFX96 Real-Time System (Bio-Rad Laboratories, Inc., Hercules, CA, USA) and the following conditions were used: $95^{\circ} \mathrm{C}$ for $5 \mathrm{~min}$; 40 cycles of $95^{\circ} \mathrm{C}$ for $30 \mathrm{sec}, 58^{\circ} \mathrm{C}$ for $30 \mathrm{sec}$ and $72^{\circ} \mathrm{C}$ for $30 \mathrm{sec}$; and $72^{\circ} \mathrm{C}$ for $10 \mathrm{~min}$. A miScript SYBR-Green PCR kit (Guangzhou RiboBio Co., Ltd., Guangzhou, China) was used to detect the expression of miR-132. The specific primer sets for miRNA-132 and U6 were purchased from GeneCopoeia, Inc. (Rockville, MD, USA) and miR-132 expression was normalized by U6. The $2^{-\Delta \Delta \mathrm{CT}}$ method was used to analyze the expression data.

ELISA determination of $M e C P 2, B D N F$ and corticosterone. Human MeCP2, BDNF and corticosterone ELISA kits (EpiQuik, Epigentek Group, Inc., Farmingdale, NY, USA) were used to determine the levels in the blood samples. According to the manufacturer's instructions, the supernatants $(100 \mu \mathrm{l})$ of the blood samples were used to measure the total protein of each sample. The samples were stored overnight at $4^{\circ} \mathrm{C}$ prior to ELISA and the supernatants were then collected. Briefly, the supernatants and antibodies were mixed and incubated in 96 -well plates at $37^{\circ} \mathrm{C}$ for $4 \mathrm{~h}$. Subsequently, the plates were washed with PBS and incubated with horseradish peroxidase-labeled anti-rabbit antibody for $30 \mathrm{~min}$ at $37^{\circ} \mathrm{C}$. Stop solutions were then added to the wells in the dark, and absorbance was measured at a wavelength of $450 \mathrm{~nm}$ using a Synergy ${ }^{\mathrm{TM}}$ Mx Microplate Reader (BioTek Instruments, Inc., Winooski, VT, USA).

Western blotting. Total protein was extracted from the hippocampi of the rats or the primary hippocampal neurons using cold radioimmunoprecipitation lysis buffer (Wuhan Boster Biological Technology., Ltd., Wuhan, China) Protein concentration was determined by a Bicinchoninic Acid Protein Assay kit (Thermo Fisher, Inc.). The protein samples were then separated by $10 \%$ SDS-PAGE (Wuhan Boster Biological Technology., Ltd.) and transferred to a nitrocellulose membrane (Wuhan Boster Biological Technology., Ltd.). The membrane was blocked in $8 \%$ non-fat dried milk in PBS for $4 \mathrm{~h}$, and was then incubated with the following primary antibodies: Rabbit monoclonal anti-MeCP2 [dilution, 1:2,000 (cat. no. 3456); Cell Signaling Technology, Inc., Danvers, MA, USA], rabbit polyclonal anti-BDNF [dilution, 1:1,000 (cat. no. ab6201); Abcam, Cambridge, UK), and mouse monoclonal anti-GAPDH [dilution, 1:3,000 (cat. no. BM1985); Wuhan Boster Biological Technology, Ltd.) overnight at $4^{\circ} \mathrm{C}$. The membrane was washed with Tris-buffered saline and Tween-20 (Wuhan Boster Biological Technology., Ltd.) and incubated with a corresponding secondary antibody for $1 \mathrm{~h}$ at $37^{\circ} \mathrm{C}$. Enhanced chemiluminescence reagent (Wuhan Boster Biological Technology, Ltd.) was used to detect the signal on the membrane. The data were analyzed by densitometry using Image-Pro Plus software 6.0 (Media Cybernetics, Inc., Rockville, MD, USA), and normalized to internal control expression.

Dual luciferase reporter assay. Wild type (wt) and mutant (mut) 3'UTRs of MeCP2 and BDNF were constructed into the $\mathrm{XbaI}$ site of the pGL3-control vector (Promega Corporation, Madison, WI, USA), downstream of the luciferase gene. All of the reporter vector construction and site-directed mutagenesis were performed by GeneCopoeia, Inc. (Guangzhou, China). For the luciferase assay, $1 \times 10^{5}$ cells were cultured in 24-well plates, until they had reached $\sim 70 \%$ confluence. Subsequently, the cells were co-transfected with miR-132 mimic, and wt or mut 3'UTR of MeCP2 or BDNF dual luciferase reporter vector, respectively. Following a $3 \mathrm{~h}$ incubation with the transfection reagent/DNA complex, the medium was refreshed with fresh medium supplemented with $10 \%$ fetal bovine serum (each from Invitrogen Life Technologies). Post-transfection (48 h), a Dual Luciferase Reporter Gene Assay kit (BioVision, Inc., Milpitas, CA, USA) was used to determine the luciferase activities of each group using a luminometer (Roche Diagnostics, Basel, Switzerland). Renilla luciferase activity was normalized to firefly luciferase activity.

Statistical analysis. Statistical analyses were performed using GraphPad Prism 5 (GraphPad Software, Inc., La Jolla, CA, USA). The data are presented as the mean \pm standard deviation. An unpaired two-tailed Student's t-test was used to analyze the results. $\mathrm{P}<0.05$ was considered to indicate a statistically significant difference.

\section{Results}

miR-132 expression is negatively correlated with MeCP2 and BDNF protein expression in peripheral blood samples of patients with MDD. The expression levels of miR-132 were detected by RT-qPCR analysis. The large panel of samples included blood samples from 30 patients with MDD and 30 normal controls. miR-132 expression was significantly increased in the peripheral blood of patients with MDD, as compared with the paired normal controls (Fig. 1A). In addition, an ELISA assay was used to measure the protein expression of MeCP2. As shown in Fig. 1B, the protein expression of $\mathrm{MeCP} 2$ was significantly decreased in the peripheral blood of patients with MDD, as compared with the normal controls. Furthermore, there was a significant decrease in BDNF expression detected in the peripheral blood of patients with MDD, as compared with the controls (Fig. 1C). The expression of miR-132 was negatively correlated with the protein expression levels of MeCP2 and BDNF, whereas MeCP2 expression was positively correlated with BDNF expression in the peripheral blood of patients with MDD (Fig. 1D-F).

MeCP2 and BDNF are downregulated, and miR-132 is upregulated in rats exposed to CUS. The CUS model is a well-established animal model of depression that mimics 
A
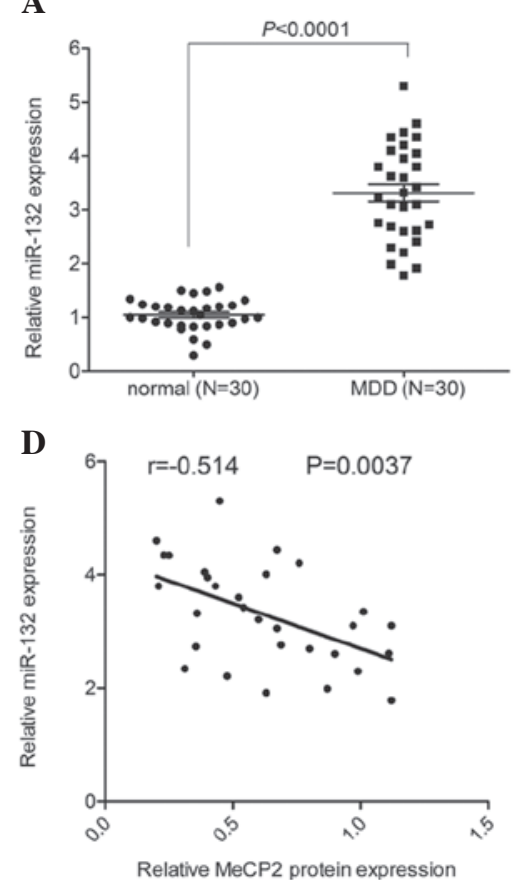

B

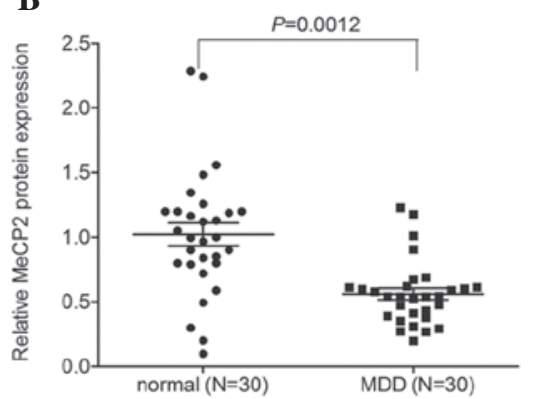

E

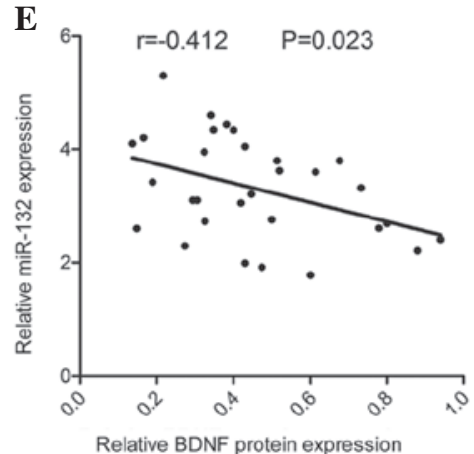

C

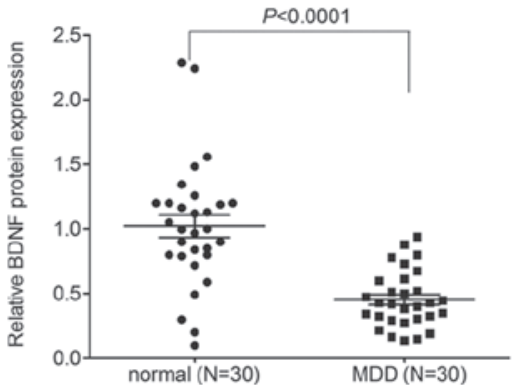

F

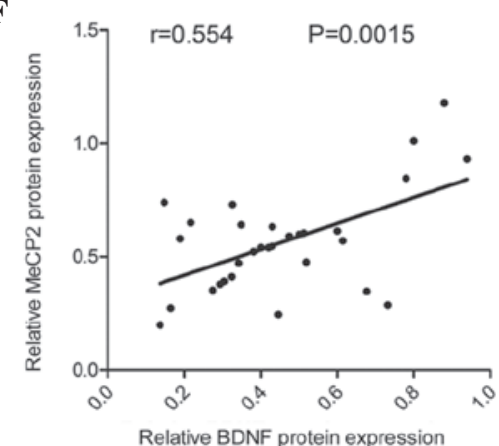

Figure 1. Correlation between miR-132 and BDNF/ MeCP2 expression in patients with MDD. (A) Relative expression levels of (A) miR-132, (B) MeCP2 and (C) BDNF in blood samples of normal subjects or patients with MDD. Correlation analysis for the expression of (D) miR-132 and MeCP2, (E) miR-132 and $\mathrm{BDNF}$, and (F) MeCP2 and BDNF. Data are presented as the mean \pm standard deviation. miR, microRNA; BDNF, brain-derived neurotrophic factor; MeCP2, methyl-CpG-binding protein 2; MDD, major depression disease.

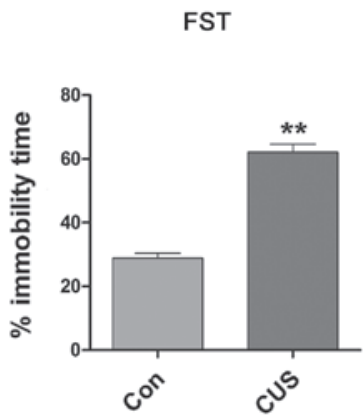

D

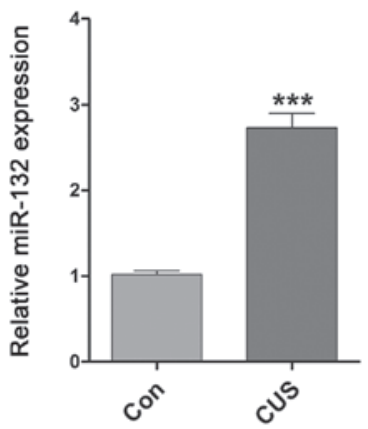

B

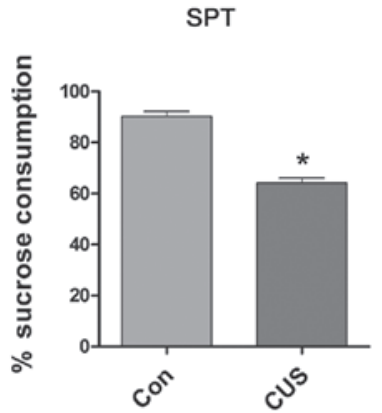

E
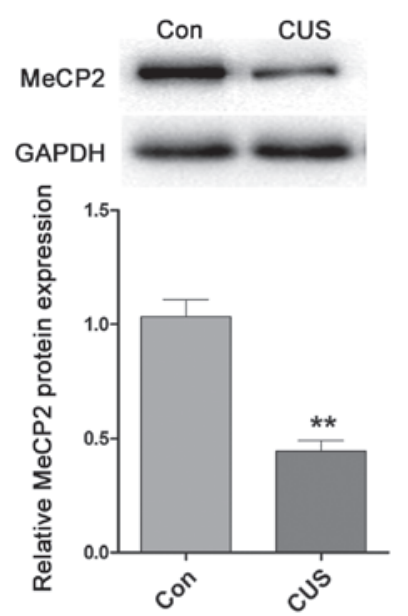

C

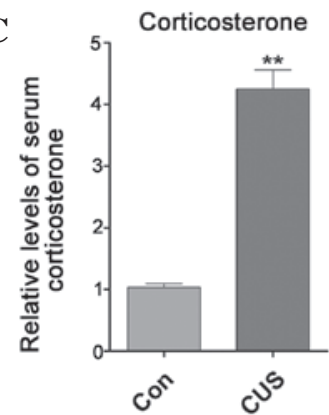

F
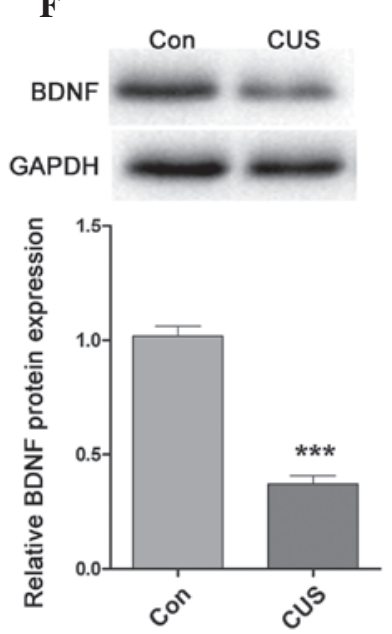

Figure 2. Expression of miR-132, BDNF and MeCP2 in CUS-exposed rats. (A) FST demonstrated increased immobility time in CUS-exposed rats, as compared with the control. (B) SPT demonstrated decreased sucrose consumption in CUS-exposed rats, as compared with the control. (C) Relative release of corticosterone in CUS-exposed and control rats, as determined by ELISA. (D) Relative expression levels of miR-132 in the hippocampi of CUS-exposed and control rats. (E) Relative expression levels of MeCP2 in the hippocampi of CUS-exposed and control rats. (F) Relative expression levels of BDNF in the hippocampi of CUS-exposed and control rats. Data are presented as the mean \pm standard deviation. ${ }^{*} \mathrm{P}<0.05,{ }^{* *} \mathrm{P}<0.01$ and ${ }^{* * *} \mathrm{P}<0.001$ vs. the control rats. miR, microRNA; BDNF, brain-derived neurotrophic factor; MeCP2, methyl-CpG-binding protein 2; CUS, chronic unpredictable stress; SPT, sucrose preference test; FST, forced swim test. 
A
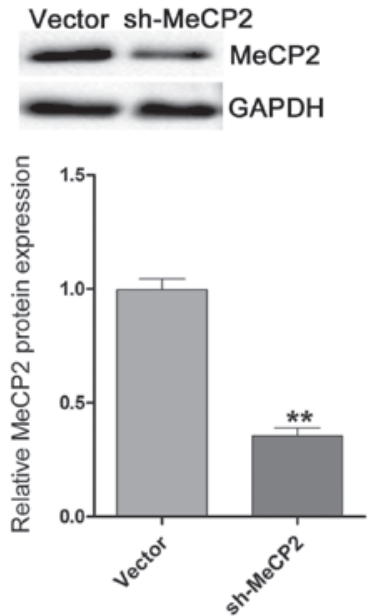

C

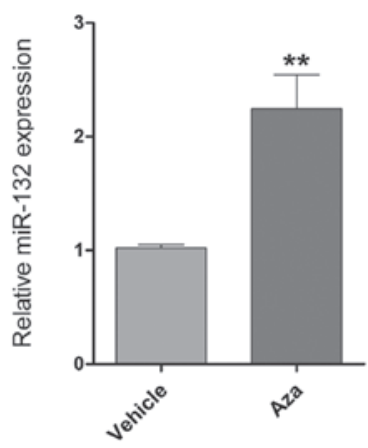

B

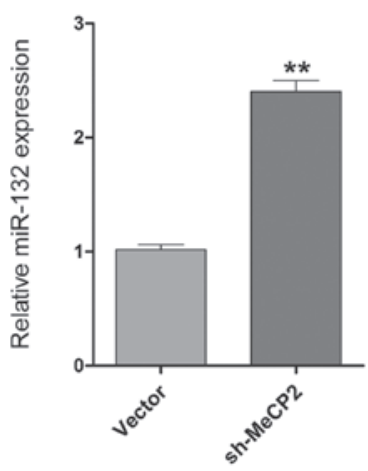

D
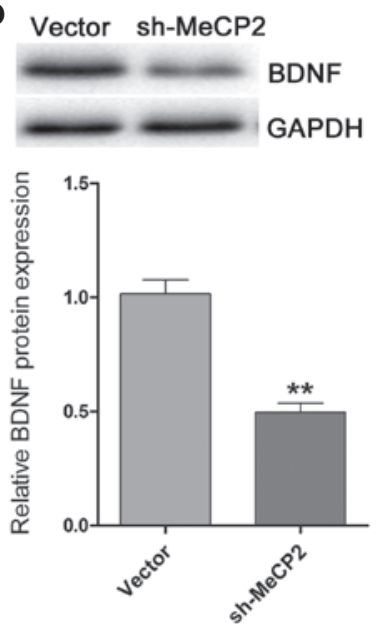

Figure 3. Knockdown of MeCP2 induces miR-132 expression and decreases BDNF expression. (A) Relative expression levels of MeCP2 in primary hippocampal neurons post-transfection. (B) miR-132 expression was induced by knockdown of MeCP2. (C) miR-132 expression was induced by treatment with the demethylation agent Aza. (D) BDNF expression was reduced by knockdown of MeCP2. Data are presented as the mean \pm standard deviation. ${ }^{* *} \mathrm{P}<0.01$ vs. the control group. $\mathrm{MeCP} 2$, methyl-CpG-binding protein 2; miR, microRNA; BDNF, brain-derived neurotrophic factor; Aza, 5-Aza-2'-deoxycytidine; sh, short hairpin RNA.

specific symptoms of human depression, such as anhedonia and learned helplessness. Following exposure to CUS, the rats exhibited high serum corticosterone levels and depressive behaviors, including increased immobility time in the FST and decreased sucrose consumption (Fig. 2A-C). Concordant with the results mentioned above, miR-132 expression was upregulated in the hippocampi of CUS-exposed rats, whereas the protein expression levels of MeCP2 and BDNF were significantly decreased (Fig. 2D-F). These results are concordant with the results from the blood samples of human patients with MDD.

MeCP 2 knockdown induces miR-132 expression and decreases $B D N F$ expression. To determine the association between $\mathrm{MeCP} 2$, miR-132 and BDNF, the expression of MeCP2 was reduced by transfecting sh-MeCP2 or a control vector, into primary hippocampal neurons. Transfection with sh-MeCP2 efficiently decreased the expression levels of MeCP2, as compared with the control vector (Fig. 3A). Furthermore,

the expression levels of miR-132 were detected. Increased expression levels of miR-132 were detected in the primary hippocampal neurons transfected with sh-MeCP2 (Fig. 3B). Furthermore, the expression of miR-132 was also induced following treatment of the primary hippocampal neurons with the demethylation agent Aza (Fig. 3C). However, MeCP2 knockdown reduced BDNF expression (Fig. 3D). These results suggest that MeCP2 is able to regulate the expression of miR-132, which may be involved in methylation.

miR-132 directly targets MeCP2 and regulates $B D N F$ expression. To investigate whether miR-132 targets the 3 'UTR of MeCP2 or BDNF, the wt 3'UTR of MeCP2 or BDNF (wt-MeCP2 or wt-BDNF) was cloned downstream to a luciferase reporter gene. In addition, a mutant 3'UTR (mut-MeCP2 or mut-BDNF) was constructed using binding site mutagenesis. The wt-MeCP2 vector was co-transfected with pre-miR-132 mimics or a scramble control into primary hippocampal neurons. The luciferase activity of pre-miR-132 and wt-MeCP2 co-transfected cells was significantly reduced, as compared with the scramble control cells (Fig. 4A). However, there were no significant changes in the luciferase activity of pre-miR-132 and wt-BDNF co-transfected cells, as compared with the scramble control cells (Fig. 4B). In addition, pre-miR-132 and anti-miR-132 were transfected into primary hippocampal neurons. As shown in Fig. 4C, the transfection efficiency was satisfactory for further analysis. Overexpression of miR-132 significantly reduced the expression levels of MeCP2, at both the mRNA and protein level, whereas downregulation of miR-132 increased the mRNA and protein expression levels of MeCP2 (Fig. 4D and E). Furthermore, the protein expression levels of BDNF were detected in the cells transfected with pre-miR-132 or anti-miR-132. The protein expression levels of BDNF were decreased following upregulation of miR-132, and increased following downregulation of miR-132 (Fig. 4F). Reduced BDNF expression was detected in the pre-miR-132 combined with sh-MeCP2 treatment group, whereas treatment with anti-miR-132 combined with sh-MeCP2 had little effect on BDNF expression (Fig. 4G). These results indicate that $\mathrm{MeCP} 2$ and miR-132 may regulate hippocampal BDNF expression in an opposite manner, suggesting that homeostatic interactions between these factors control hippocampal BDNF expression.

\section{Discussion}

The symptoms of depression have been well characterized, and include anhedonia and learned helplessness; however, the underlying pathogenic mechanism remains unclear. The present study demonstrated that miR-132 was significantly increased in the peripheral blood of patients with MDD, as compared with the normal subjects. The expression levels of miR-132 were shown to be negatively correlated with the protein expression levels of MeCP2 and BDNF. In addition, in a rat model of CUS-induced depression, miR-132 expression was upregulated in the hippocampi of CUS-exposed rats, whereas the protein expression levels of MeCP2 and BDNF were significantly decreased. Since it is difficult to determine BDNF levels directly from the brain of patients, an increased effort has been made regarding the measurement of BDNF 
A

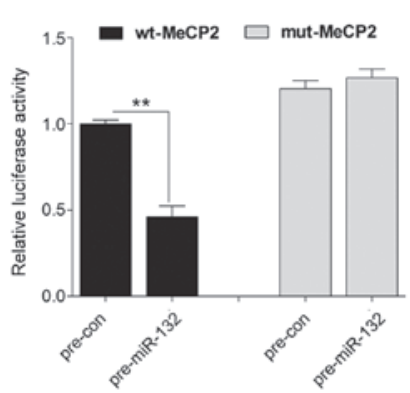

D

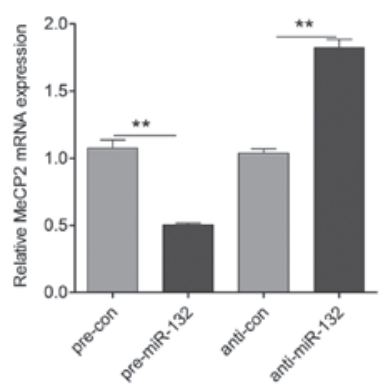

G
B

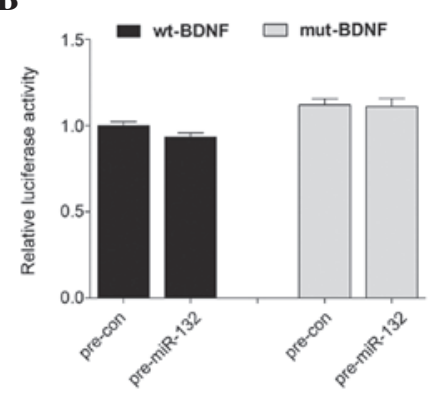

$\mathbf{E}$
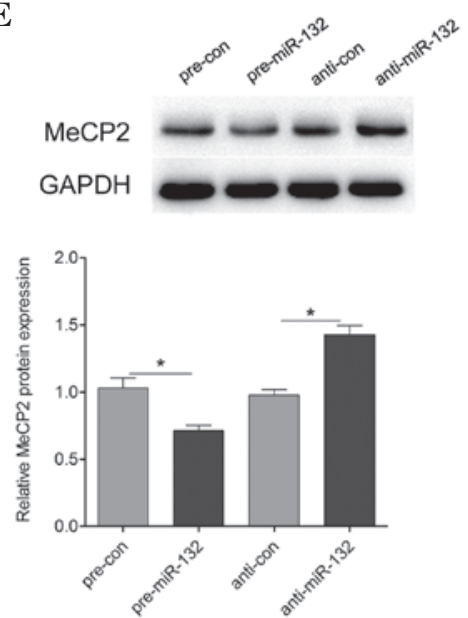

C

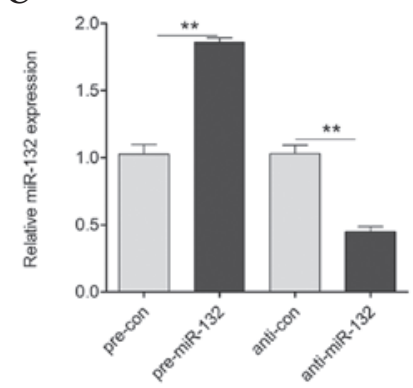

$\mathbf{F}$
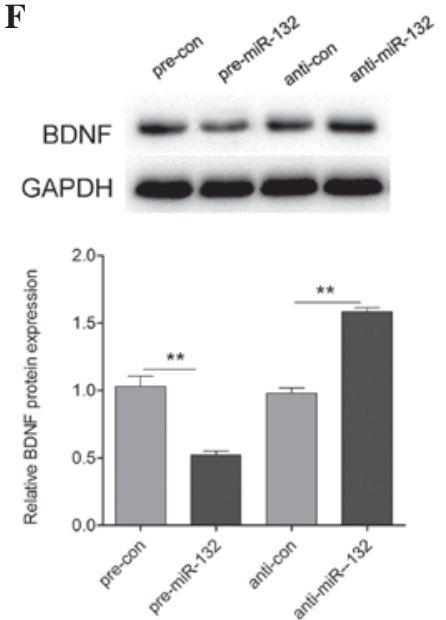
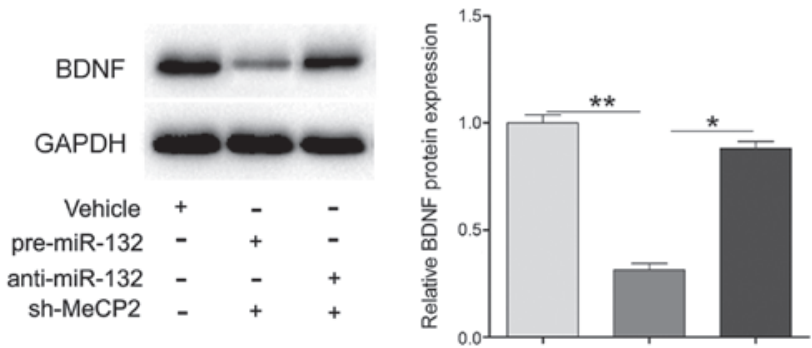

Figure 4. miR-132 directly targets the 3' UTR of MeCP2. (A) The repression of luciferase activity by wt-MeCP2 3'UTR was dependent on miR-132. Mut-MeCP2 3'UTR abrogated miR-132 mediated repression of luciferase activity. (B) Neither wt-BDNF 3'UTR nor mut-BDNF 3'UTR affected luciferase activity. (C) Expression levels of miR-132 following pre-miR-132 or anti-miR-132 treatment in primary hippocampal neurons, as detected by RT-qPCR. (D) Expression levels of MeCP2 mRNA after pre-miR-132 or anti-miR-132 treatment, as detected by RT-qPCR. (E) Western blotting detected the protein expression levels of $\mathrm{MeCP} 2$ after pre-miR-132 or anti-miR-132 treatment. (F) Western blotting detected the protein expression levels of BDNF in primary hippocampal neurons after pre-miR-132 or anti-miR-132 treatment. (G) Western blotting detected the protein expression levels of BDNF in primary hippocampal neurons after the indicated treatments. Data are presented as the mean \pm standard deviation. "P $<0.05$ and ${ }^{* *} \mathrm{P}<0.01$ vs. the control groups. miR, microRNA; UTR, untranslated region; MeCP2, methyl-CpG-binding protein 2; wt, wild type; mut, mutated; BDNF, brain-derived neurotrophic factor; RT-qPCR, reverse transcription-quantitative polymerase chain reaction.

levels in peripheral blood, as the BDNF levels in the brain of patients with mood disorders would be reliably reflected by levels in the peripheral blood. It has previously been reported that serum BDNF levels were decreased, whereas the levels of miR-132 were increased, in patients with depression as compared with those of healthy controls (7). Furthermore, serum BDNF levels are increased following treatment with antidepressant agents (21), thus suggesting that serum BDNF levels may be used as a potential biomarker for measuring depression status and the efficacy of antidepressant treatment. In addition, previous studies have shown that BDNF gene polymorphisms are associated with depression-related traits $(22,23)$. In an animal model of depression, decreased
BDNF levels in the brain and serum have been well demonstrated (24), suggesting that BDNF may have a critical role as a susceptibility gene in depression. Therefore, controlling BDNF levels may be an important factor for the management of depression.

MeCP2 levels are closely correlated with BDNF (25); however, the underlying dynamics of this complex relationship in the brain are not fully clear. Numerous studies have identified BDNF as a target that may be regulated by MeCP2, which is relevant to the pathogenesis of MDD (26-28). By binding to the BDNF promoter, $\mathrm{MeCP} 2$ directly modulates BDNF expression in an activity-dependent manner (29). Neuronal activity rapidly induces the dissociation of $\mathrm{MeCP} 2$ from the 
BDNF promoter, which enhances the expression of BDNF in response to neuronal activity. Mecp2-deficient mice have been shown to exhibit reduced BDNF mRNA and protein expression in various brain regions, including the hippocampus (30). In addition, reduced overall neuronal activity caused by $\mathrm{MeCP} 2$ deficiency is hypothesized to contribute to BDNF downregulation, whereas BDNF overexpression rescued certain functional deficits observed in MeCP2 mutants and extended their lifespan (31). Furthermore, MeCP2 overexpression in cultured mouse cortical neurons results in increased BDNF expression (18). A previous study demonstrated that phosphorylation of MeCP2 at serine 421 has a critical role in regulating the development of hippocampal dendritic spines and the mature form of excitatory synapses (32). Concordant with the findings of previous studies, the present study demonstrated that reduced $\mathrm{BDNF}$ and $\mathrm{MeCP} 2$ protein expression levels were detected in CUS-exposed rats that exhibited depressive-like behavior. Furthermore, knockdown of MeCP2 in primary hippocampal neurons decreased BDNF protein expression levels. These results indicated that hippocampal MeCP2 may directly regulate BDNF expression in CUS-exposed rats, which may contribute to neuronal activity and survival.

Knockdown of MeCP2 in primary hippocampal neurons not only decreased BDNF protein expression levels, but also induced miR-132 expression. In addition, treatment with Aza, a demethylation agent, induced miR-132 expression in primary hippocampal neurons. These results indicated that miR-132 expression may be regulated by $\mathrm{MeCP} 2$ via an epigenetic mechanism. A previous study demonstrated that in patients with depression there is a significant positive correlation between Self-Rating Depression Scale scores and the expression of miR-132, and an inverse correlation between serum BDNF levels and miR-132 levels in depression (7). A previous study reported on miRNAs associated with the sensitivity of human lymphoblastoid cell lines to selective serotonin reuptake inhibitors. It was demonstrated that miR-212 and miR-132 were differentially expressed between two human lymphoblastoid cell lines, which exhibited high or low sensitivities to paroxetine (33). Furthermore, specific overexpression of miR-132 in the perirhinal cortex impaired the short-term recognition memory of rats (34). In addition, footshock stress and predator scent stress induced a long-lasting hippocampal elevation of miR-132 expression (35). These results suggested a role of miR-132 in the neuronal mechanisms underlying the symptoms of depression.

Furthermore, in the present study it was demonstrated that miR-132 was able to regulate $\mathrm{MeCP} 2$ at the mRNA and protein level by directly targeting its 3'UTR. However, it could not directly target BDNF, although BDNF protein expression levels were affected by miR-132. As mentioned above, MeCP2 expression was positively correlated, whereas miR-132 expression was negatively correlated, with BDNF levels in patients with MDD. In addition, in CUS-exposed rats, hippocampal MeCP2 and BDNF expression was decreased, whereas hippocampal miR-132 expression was increased. Furthermore, downregulated expression of BDNF, induced by MeCP2 knockdown, was enhanced by miR-132 mimics and rescued by miR-132 inhibitors. These results demonstrated that MeCP2-miR-132 homeostatic interactions may control the hippocampal BDNF levels, which are associated with depression. BDNF can be synthesized locally in the hippocampus in an activity-dependent manner, and unpredictable and persistent stress can reduce local BDNF production. The results of the present study suggested that unpredictable and persistent stress was able to decrease the de novo production of BDNF in the hippocampus. Furthermore, downregulation of MeCP2 may result in loss of the inhibitory capacity of the repressors of BDNF transcription, such as RE1 silencing transcription factor (36). In the same manner, miR-132 may decrease hippocampal BDNF levels by silencing MeCP2 expression. A recent study demonstrated that $\mathrm{MeCP} 2$ serves as a necessary co-activator of cAMP response element-binding protein activity at the BDNF promoter (37). In this way, MeCP2 levels may determine the stimulatory effects of CREB signaling on BDNF production in the hippocampus. The present study demonstrated that MeCP2 and miR-132 have opposite effects on hippocampal BDNF levels, suggesting that homeostatic interactions between MeCP2 and miR-132 may have a key role in depression.

In conclusion, the present study highlights the interaction between MeCP2 and miR-132 in regulating hippocampal BDNF levels, suggesting that miR-132 may be a key factor in controlling stress-induced hippocampal neuroplasticity and neuronal survival in depression.

\section{References}

1. Dwivedi Y: Emerging role of microRNAs in major depressive disorder: Diagnosis and therapeutic implications. Dialogues Clin Neurosci 16: 43-61, 2014.

2. Mouillet-Richard S, Baudry A, Launay JM and Kellermann O: MicroRNAs and depression. Neurobiol Dis 46: 272-278, 2012.

3. Sala M, Perez J, Soloff P, Ucelli di Nemi S, Caverzasi E, Soares JC and Brambilla P: Stress and hippocampal abnormalities in psychiatric disorders. Eur Neuropsychopharmacol 14: 393-405, 2004.

4. Li M, Fu Q, Li Y, Li S, Xue J and Ma S: Emodin opposes chronic unpredictable mild stress induced depressive-like behavior in mice by upregulating the levels of hippocampal glucocorticoid receptor and brain-derived neurotrophic factor. Fitoterapia 98: 1-10, 2014.

5. Maripuu M, Wikgren M, Karling $\mathrm{P}$, Adolfsson $\mathrm{R}$ and Norrback KF: Relative hypo- and hypercortisolism are both associated with depression and lower quality of life in bipolar disorder: A cross-sectional study. PLoS One 9: e98682, 2014.

6. Réus GZ, Abelaira HM, Stringari RB, Fries GR, Kapczinski F and Quevedo J: Memantine treatment reverses anhedonia, normalizes corticosterone levels and increases BDNF levels in the prefrontal cortex induced by chronic mild stress in rats. Metab Brain Dis 27: 175-182, 2012.

7. Li YJ, Xu M, Gao ZH, Wang YQ, Yue Z, Zhang YX, Li XX, Zhang C, Xie SY and Wang PY: Alterations of serum levels of BDNF-related miRNAs in patients with depression. PLoS One 8: e63648, 2013

8. Bai M, Zhu X, Zhang Y, Zhang S, Zhang L, Xue L, Yi J, Yao S and Zhang X: Abnormal hippocampal BDNF and miR-16 expression is associated with depression-like behaviors induced by stress during early life. PLoS One 7: e46921, 2012.

9. Bahi A, Chandrasekar V and Dreyer JL: Selective lentiviral-mediated suppression of microRNA124a in the hippocampus evokes antidepressants-like effects in rats. Psychoneuroendocrinology 46: 78-87, 2014.

10. O'Connor RM, Grenham S, Dinan TG and Cryan JF: microRNAs as novel antidepressant targets: Converging effects of ketamine and electroconvulsive shock therapy in the rat hippocampus. Int J Neuropsychopharmacol 16: 1885-1892, 2013.

11. Dell'Osso B, D'Addario C, Carlotta Palazzo M, Benatti B, Camuri G, Galimberti D, Fenoglio C, Scarpini E, Di Francesco A, Maccarrone M and Altamura AC: Epigenetic modulation of BDNF gene: Differences in DNA methylation between unipolar and bipolar patients. J Affect Disord 166: 330-333, 2014. 
12. Zucchi FC, Yao Y, Ward ID, Ilnytskyy Y, Olson DM, Benzies K, Kovalchuk I, Kovalchuk O and Metz GA: Maternal stress induces epigenetic signatures of psychiatric and neurological diseases in the offspring. PLoS One 8: e56967, 2013.

13. Murgatroyd C, Wu Y, Bockmühl Y and Spengler D: Genes learn from stress: How infantile trauma programs us for depression. Epigenetics 5: 194-199, 2010.

14. Forlani G, Giarda E, Ala U, Di Cunto F, Salani M, Tupler R, Kilstrup-Nielsen $\mathrm{C}$ and Landsberger N: The MeCP2/YY1 interaction regulates ANT1 expression at 4q35: Novel hints for Ret syndrome pathogenesis. Hum Mol Genet 19: 3114-3123, 2010.

15. Hutchinson AN, Deng JV, Cohen S and West AE: Phosphorylation of MeCP2 at Ser421 contributes to chronic antidepressant action. J Neurosci 32: 14355-14363, 2012.

16. Murgatroyd C, Patchev AV, Wu Y, Micale V, Bockmühl Y, Fischer D, Holsboer F, Wotjak CT, Almeida OF and Spengler D: Dynamic DNA methylation programs persistent adverse effects of early-life stress. Nat Neurosci 12: 1559-1566, 2009.

17. Wada R, Akiyama Y, Hashimoto Y, Fukamachi H and Yuasa Y: miR-212 is downregulated and suppresses methyl-CpG-binding protein $\mathrm{MeCP} 2$ in human gastric cancer. Int J Cancer 127: 1106-1114, 2010.

18. Klein ME, Lioy DT, Ma L, Impey S, Mandel G and Goodman RH: Homeostatic regulation of $\mathrm{MeCP} 2$ expression by a CREB-induced microRNA. Nat Neurosci 10: 1513-1514, 2007.

19. Im HI, Hollander JA, Bali P and Kenny PJ: MeCP2 controls BDNF expression and cocaine intake through homeostatic interactions with microRNA-212. Nat Neurosci 13: 1120-1127, 2010.

20. Luo YW, Xu Y, Cao WY, Zhong XL, Duan J, Wang XQ, Hu ZL, Li F, Zhang JY, Zhou M, Dai RP and Li CQ: Insulin-like growth factor 2 mitigates depressive behavior in a rat model of chronic stress. Neuropharmacology 89: 318-324, 2015.

21. Munno D, Sterpone S, Fania S, Cappellin F, Mengozzi G, Saroldi M, Bechon E and Zullo G: Plasma brain derived neurotrophic factor levels and neuropsychological aspects of depressed patients treated with paroxetine. Panminerva Med 55: 377-384, 2013.

22. Yu H, Wang DD, Wang Y, Liu T, Lee FS and Chen ZY: Variant brain-derived neurotrophic factor Val66Met polymorphism alters vulnerability to stress and response to antidepressants. J Neurosci 32: 4092-4101, 2012.

23. Carballedo A, Amico F, Ugwu I, Fagan AJ, Fahey C, Morris D, Meaney JF, Leemans A and Frodl T: Reduced fractional anisotropy in the uncinate fasciculus in patients with major depression carrying the met-allele of the Val66Met brain-derived neurotrophic factor genotype. Am J Med Genet B Neuropsychiatr Genet 159B: 537-548, 2012.

24. Valvassori SS, Budni J, Varela RB and Quevedo J: Contributions of animal models to the study of mood disorders. Rev Bras Psiquiatr 35 (Suppl 2): S121-S131, 2013.

25. Xu X, Kozikowski AP and Pozzo-Miller L: A selective histone deacetylase-6 inhibitor improves BDNF trafficking in hippocampal neurons from Mecp2 knockout mice: Implications for Rett syndrome. Front Cell Neurosci 8: 68, 2014.
26. Khoshnan A and Patterson PH: Elevated IKK $\alpha$ accelerates the differentiation of human neuronal progenitor cells and induces MeCP2-dependent BDNF expression. PLoS One 7: e41794, 2012.

27. Cortés-Mendoza J, Díaz de León-Guerrero S, Pedraza-Alva G and Pérez-Martínez L: Shaping synaptic plasticity: the role of activity-mediated epigenetic regulation on gene transcription. Int J Dev Neurosci 31: 359-369, 2013.

28. Lv J, Xin Y,Zhou W and Qiu Z. The epigenetic switches for neural development and psychiatric disorders. J Genet Genomics 40: 339-346, 2013.

29. Li H, Zhong X, Chau KF, Williams EC and Chang Q: Loss of activity-induced phosphorylation of $\mathrm{MeCP} 2$ enhances synaptogenesis, LTP and spatial memory. Nat Neurosci 14: 1001-1008, 2011.

30. Li W, Calfa G,Larimore J and Pozzo-Miller L: Activity-dependent BDNF release and TRPC signaling is impaired in hippocampal neurons of Mecp2 mutant mice. Proc Natl Acad Sci USA 109: 17087-17092, 2012.

31. Chang Q, Khare G, Dani V, Nelson S and Jaenisch R: The disease progression of Mecp2 mutant mice is affected by the level of BDNF expression. Neuron 49: 341-348, 2006.

32. Zhou Z, Hong EJ, Cohen S, Zhao WN, Ho HY, Schmidt L, Chen WG, Lin Y, Savner E, Griffith EC, et al: Brain-specific phosphorylation of MeCP2 regulates activity-dependent Bdnf transcription, dendritic growth, and spine maturation. Neuron 52: 255-269, 2006

33. Oved K, Morag A, Pasmanik-Chor M, Oron-Karni V, Shomron N, Rehavi M, Stingl JC and Gurwitz D: Genome-wide miRNA expression profiling of human lymphoblastoid cell lines identifies tentative SSRI antidepressant response biomarkers. Pharmacogenomics 13: 1129-1139, 2012.

34. Scott HL, Tamagnini F, Narduzzo KE, Howarth JL, Lee YB, Wong LF, Brown MW, Warburton EC, Bashir ZI and Uney JB: MicroRNA-132 regulates recognition memory and synaptic plasticity in the perirhinal cortex. Eur J Neurosci 36: 2941-2948, 2012.

35. Shaltiel G, Hanan M, Wolf Y, Barbash S, Kovalev E, Shoham S and Soreq H: Hippocampal microRNA-132 mediates stress-inducible cognitive deficits through its acetylcholinesterase target. Brain Struct Funct 218: 59-72, 2013.

36. Abuhatzira L, Makedonski K, Kaufman Y, Razin A and Shemer R: MeCP2 deficiency in the brain decreases BDNF levels by REST/CoREST-mediated repression and increases TRKB production. Epigenetics 2: 214-222, 2007.

37. Chahrour M, Jung SY, Shaw C, Zhou X, Wong ST, Qin J and Zoghbi HY: MeCP2, a key contributor to neurological disease, activates and represses transcription. Science 320: 1224-1229, 2008. 